\title{
Long non-coding RNA LINC00174 promotes glycolysis and tumor progression by regulating miR-152-3p/SLC2A1 axis in glioma
}

\author{
Jian Shi ${ }^{\dagger}$, Yang Zhang ${ }^{\dagger}$, Bing Qin, Yongjie Wang and Xiangdong Zhu ${ }^{*}$
}

\begin{abstract}
Background: Long non-coding RNA plays a crucial role in the occurrence and progression of glioma. We aimed to explore the function of LINC00174 in cell proliferation, apoptosis, migration, invasion and glycolysis of glioma cells, and investigate the molecular mechanism involved.

Methods: LINC00174 expression in glioma tissues and peritumoral brain edema (PTBE) tissues was examined by RTqPCR and in situ hybridization. The CCK-8, TUNEL, wound healing, transwell, and ELISA assays were performed to identify the effects of LINC00174 knockdown on cell viability, apoptosis, migration, invasion, and glycolysis, respectively. RNA immunoprecipitation, dual-luciferase reporter, RNA pull down, and western blot assays were performed to explore the molecular mechanisms of LINC00174 in glioma cells. A nude mouse xenograft model was used to investigate the role of LINC00174 in xenograft glioma growth.

Results: LINC00174 was overexpressed in glioma tissues and cell lines. LINC00174 knockdown inhibited cell proliferation, migration, invasion and glycolysis of glioma cells, and LINC00174 exerted a tumorigenesis role. LINC00174 could interact with miR-152-3p/SLC2A1 axes. The miR-152-3p inhibitor or the SLC2A1 overexpression could rescue the anti-tumor effect of LINC00174 knockdown on glioma cells. Moreover, downregulation of LINC00174 also inhibited tumor volume and delayed the tumor growth in vivo.
\end{abstract}

Conclusion: LINC00174 accelerated carcinogenesis of glioma via sponging miR-1523-3p and increasing the SLC2A1 expression, which could be considered as a molecular target for glioma diagnosis and therapy.

Keywords: LINC00174, Glioma, SLC2A1, Glycolysis, miR-152-3p

\section{Highlights}

LINC00174 was overexpressed in glioma.

LINC00174 predicted an unfavorable prognosis in glioma patients.

LINC00174 promoted glycolysis and tumor progression by targeting miR-152-3p/SLC2A1 axes.

\footnotetext{
* Correspondence: zhuxd@126.com

${ }^{\dagger}$ Dr Jian Shi and Dr Yang Zhang contributed equally to the study, and they are co-first authors.

Department of Neurosurgery, The Second Affiliated Hospital of Zhejiang University School of Medicine, No. 88 Jiefang Road, Hangzhou 310000, China
}

\section{Backgroud}

Glioma is the most common primary brain tumor, which has the characteristics of high morbidity, high recurrence rate and high mortality, and seriously endangers people's health [1]. It originates from astrocyte and oligodendrocyte, which are called astrocytoma and oligodendroglioma respectively [2]. The annual incidence of glioma in the population is $6.13 / 100,000$ [3]. Although surgery combined with radiotherapy and chemotherapy can partly delay the recurrence time and prolong the survival time of glioma patients, the curative effect on some glioma patients with high malignancy is still unsatisfied. The median survival time of patients with WHO grade III degenerative astrocytoma and WHO grade IV 
pleomorphic glioblastoma was only 2 years and 1 year, respectively, even after surgery combined with radiotherapy and chemotherapy [3]. In the twentieth century, with the development of molecular biology and oncogenetics, people have a better understanding of the genetic characteristics of glioma, which provides a new idea for the subsequent gene therapy of glioma. Therefore, at present, the international community is focusing on the search for effective gene markers of glioma, hoping to be conducive to early intervention treatment and early prognosis judgment of glioma [4-6].

Long-chain non-coding RNA (lncRNA) is a kind of RNA with a length range of 200 nucleotides to 100,000 bases, which lacks an effective open reading framework and is transcribed by RNA polymerase II without protein coding function [7]. The main regulation modes of lncRNA include: (1) as a transcription regulator, interfering with the binding of transcription factors to promoters, interfering with gene transcription and chromatin remodeling; (2) as a regulator, influencing the transcription of target genes, up-regulating or down-regulating the expression of target genes; (3) binding to proteins through chaperone and regulating subcellular localization of proteins; and (4) binding to transcription to inhibit replication [8-10]. Through these pathways, IncRNA can play a variety of biological functions at the transcriptional level, post-transcriptional level and epigenetic level. Recent studies have shown that abnormal expression of IncRNA may affect the occurrence and development of glioma. A lot of lncRNAs have been reported to accelerate the tumorigenesis of glioma, including MALAT1 [11], SNHG16 [12], UCA1 [13]. According to the The Cancer Genome Atlas (TCGA) data analysis, we found the expression level of lncRNA LINC00174 was up-regulated in glioma tissue samples. Shen et al. reported that increased expression of LINC00174 was observed in colorectal cancer (CRC) tissues and cells, and LINC00174 indicated the poor prognosis of CRC patients [14]. However, the biological function of LINC00174 in the pathogenesis and development of glioma still remains unknown. Starbase predicted that LINC00174 could interact with miR-152$3 p$, which acts as an anti-cancer role in glioma $[15,16]$. According to the prediction of Targetscan website, it was found that miR-152-3p can directly target Solution vector family 2 promotes glucose transporter 1 (SLC2A1), also known as glucose transporter 1 (GLUT1), which is a key protein in the energy metabolism pathway of cells [17]. SLC2A1 is overexpressed in several different types of cancer, including liver cancer, lung cancer, endometrial cancer, oral cancer, breast cancer, gastric cancer and glioma [18-21]. These observations suggest that SLC2A1 may be one of the driving genes in tumors. However, it is not clear whether LINC00174 can regulate the expression of microRNA-152-3p and SLC2A1 to play a role in glioma.
Therefore, the objective of this study is to investigate whether LINC00174 promotes glycolysis and glioma progression by regulating the miR-152-3p/SLC2A1 axes.

In the present study, we explored the expression of LINC00174 in glioma tissues and normal tissues. The effect of LINC00174 on glioma progression was studied, and the underlying molecular mechanism by which LINC00174 regulated glioma cell phenotype was also investigated.

\section{Materials and methods \\ Clinical tissue specimens}

Forty-five paired brain glioma specimens and peritumoral brain edema (PTBE) tissues were collected from surgical tumor resections performed at The Second Affiliated Hospital of Zhejiang University School of Medicine (Hangzhou, China). Samples were collected between 2014 and 2017. Tissues were snap-frozen in liquid nitrogen and stored at $-80^{\circ} \mathrm{C}$ for subsequent analysis. All tissues were obtained with written informed consent from each patient. The present study conformed to the ethical guidelines of the 1975 Declaration of Helsinki and was approved by the Institutional Ethical Review Committee of The Second Affiliated Hospital of Zhejiang University School of Medicine.

\section{Cell lines and cell culture}

Human glioma cell lines (U251, LN229, H4, SW1783, and A172) and human embryonic kidney cell line HEK-293 T were purchased from Shanghai Institutes for Biological Sciences Cell Resource Center (Shanghai, China), while normal human astrocytes (NHAs) were purchased from Sciencell Research Laboratories (Carlsbad, CA, USA). HEK$293 \mathrm{~T}$ cells were cultured in Dulbecco's modified eagle medium (DMEM, Life Technologies, Carlsbad, CA) supplemented with glucose and 10\% fetal bovine serum (FBS; Life Technologies), glioma cells were cultured in Dulbecco's modified eagle medium/F12 mixed medium supplemented with 10\% FBS, and NHAs were cultured in astrocyte medium (Life Technologies). All cells were cultured at $37^{\circ} \mathrm{C}$ in a humidified incubator containing $5 \% \mathrm{CO}_{2}$.

\section{RNA isolation and reverse transcription-quantitative polymerase chain reaction (RT-qPCR)}

Total cellular RNA was extracted using TRIzol reagent (Invitrogen, Thermo Fisher Scientifc, Inc.), according to the manufacturer's protocol. For miRNA expression analysis, RT-qPCR was carried out by using the TaqMan MicroRNA Reverse Transcription kit, TaqMan Universal PCR Master Mix (Applied Biosystems; Thermo Fisher Scientific, Inc.). Relative miRNA expression levels were calculated as $2^{-[(\mathrm{Ct}}$ of miRNA $\left.)-\left(\begin{array}{lll}\mathrm{Ct} & \text { of } & \mathrm{U}\end{array}\right)\right]$ after normalization to the expression of small nuclear RNA U6. Primers (RiboBio) used for stem-loop reverse- 
transcription PCR of miR-152-3p and U6 were as follows: miR-152-3p forward, 5'-AGGGTCAGTGCATG ACAGA-3' and reverse, 5'-TACCAACCAACCCACTCA CT-3'; U6 forward, 5'-CGGGTGCTCGCTTCGCAGC-3' and reverse, 5'-CCA GTGCAGGGTCCGAGGT-3'. For LINC00174 and SLC2A1 expression analysis, RT-qPCR was performed by using the TaqMan High-Capacity cDNA Reverse Transcription Kit, TaqMan Fast PCR Master Mix (Applied Biosystems; Thermo Fisher Scientific, Inc.) according to the manufacturer's instructions with corresponding primers: LINC00174 forward, 5'-GGCCCAACACTTCC CTCAAA-3' and reverse, 5'-CAGGGAGAAACGAC CTGGAG-3'; SLC2A1 forward, 5'-AAGGTGATCGAGGA GTTCTACA-3' and reverse, 5' - ATGCCCCCAACAGAAA AGATG-3'; $\beta$-actin forward, 5'-TCCTCTGACTTCAA CAGCGACAC-3' and reverse, 5'-CACCCTGTTGCTGT AGCCAAATTC-3'. Gene expression levels were normalized to $\beta$-actin expression and were calculated as $2^{-[(\mathrm{Ct} \text { of }}$ GENES) - (Ct of $\beta$-actin)].

\section{Cell transfection}

Two small interfering RNAs (siRNA) targeting LINC00174 (siLINC00174\#1, and siLINC00174\#2), and negative control RNAs (siNC) were generated in pLKO.1. Plasmid constructs were transfected into cells at 70-90\% confluency using Lipofectamine 2000 (Invitrogen) and were then transfected again $24 \mathrm{~h}$ later. The siRNA targeting SLC2A1 (siSLC2A1), miR-152-3p mimics, inhibitors, and relative controls were obtained from GenePharma Co., Ltd. (Shanghai, China). Glioma cell transfection was conducted using Lipofectamine 2000 (Invitrogen) at a final concentration of $50 \mathrm{nM}$. To overexpress LINC00174, glioma cells were transfected with pcDNA3.1-LINC00174 using Lipofectamine 2000. After an additional $24 \mathrm{~h}$, the transfected cells were collected and processed for further studies.

\section{Cell Counting Kit-8 (CCK-8) assay}

Glioma cells $\left(1 \times 10^{5}\right.$ cells per well $)$ were seeded in 96-well plates and cultured for $24 \mathrm{~h}$ prior to analysis of cell proliferation using the CCK-8 (Dojindo Molecular Technologies, Gaithersburg, USA) assay. Cells were then cultured for a further 24, 48, or $72 \mathrm{~h}$. Subsequently, all cells were incubated with $10 \mu \mathrm{L}$ of CCK-8 solution at $37^{\circ} \mathrm{C}$ for $4 \mathrm{~h}$. To obtain cell growth curves, plates were read at $450 \mathrm{~nm}$ using a microplate spectrophotometer (Thermo Fisher Scientifc, Inc.). All experiments were performed in triplicate.

\section{TUNEL}

An in-situ cell death detection kit (Roche, Basel, Switzerland) was used to measure cell apoptosis. Briefly, cells were blocked with $\mathrm{H}_{2} \mathrm{O}_{2}$ (3\% in methanol) for $5 \mathrm{~min}$ and then labeled with TdT labeling reaction mix for $1 \mathrm{~h}$ at $37^{\circ} \mathrm{C}$. Nuclei exhibiting DNA fragmentation were visualized with 3,3'-diaminobenzidine (DAB) for $15 \mathrm{~min}$ and observed under a light microscope (Olympus Corporation,, Tokyo, Japan).

\section{Cell migration and invasion assay}

Cell migration was evaluated using a wound-healing assay. In brief, $48 \mathrm{~h}$ after transfection, glioma cells were cultured in 6-well plates $\left(5 \times 10^{4}\right.$ cells per well). After reaching 90 95\% confluence, the monolayer of cells was scratched with a sterile plastic micropipette tip and cells were then cultured under standard conditions for $24 \mathrm{~h}$. Following several washes, recovery of the wound was observed and imaged using an X71 inverted microscope (Olympus Corporation).

A transwell invasion assay was performed to assess cell invasion. Transfected cells $\left(1 \times 10^{5}\right)$ were seeded into the upper chamber of Matrigel-coated inserts containing serum-free medium. Medium supplemented with 10\% FBS (Life Technologies) was added to the lower chamber as a chemoattractant. Cells were then allowed to invade for $48 \mathrm{~h}$ at $37^{\circ} \mathrm{C}$ with $5 \% \mathrm{CO}_{2}$. Cells that invaded the lower chamber of the filter were fixed in $70 \%$ ethanol for $30 \mathrm{~min}$ and stained with $0.1 \%$ crystal violet for $10 \mathrm{~min}$ at $25^{\circ} \mathrm{C}$. The number of cells that migrated to the lower chamber was counted in five randomly selected fields under an X71 inverted microscope.

\section{Glucose uptake and lactate production assay}

Glioma cells were cultured in glucose-free DMEM for $16 \mathrm{~h}$, and then incubated with high-glucose DMEM under normoxic conditions for an additional $24 \mathrm{~h}$. Culture medium was then removed, and intracellular glucose levels were measured using a fluorescence-based glucose assay kit (BioVision, Milpitas, California, USA) according to the manufacturer's instructions. Lactate levels were measured using a lactate oxidase-based colorimetric assay read at $540 \mathrm{~nm}$ according to the manufacturer's instructions (Beyotime, Wuxi, China) and normalized to cell number.

\section{Bioinformatics analysis}

The target miRNAs of LINC00174 were predicted via computational algorithms, including starbase (http://starbase. sysu.edu.cn) and miRanda (http://www.microrna.org). The highest-ranked predicted target of LINC00174 was miR152-3p. To identify genes targeted by miR-152-3p, we used the online programs, TargetScan (http://www.targetscan. org/) and miRanda (http://www.microrna.org). From the list of target genes obtained, all genes likely to contribute to glioma progression were extracted. The 3'-UTR of SLC2A1 was predicted to have miR-152-3p-binding sites.

\section{Luciferase reporter assay}

To identify the LINC00174- and SLC2A1-binding sites in the miR-152-3p promoter, miR-152-3p promoter reporter 
constructs containing either wild-type, mutated LINC00174-binding sites, or mutated SLC2A1-binding sites were transfected with pRL-SV40 Renilla luciferase vectors into HEK293T cells using the LT1 Transfection Reagent (Mirus, Madison, WI, USA). Luciferase assays were performed using the Dual Luciferase Reporter Assay System (Promega, Madison, WI, USA) $48 \mathrm{~h}$ after transfection. Transfections were performed in triplicate, and measurements from transfections were analyzed after normalization to firefly luciferase activity.

\section{RNA pull-down assay}

Biotinylated RNAs were transcribed using Biotin RNA Labeling Mix (Roche) and T7 polymerase (Promega) and subsequently treated with RNase-free DNase I (Promega) and RNeasy Mini Kit (Qiagen). Next, magnetic beads were added to each binding reaction sample and incubated at room temperature. Finally, the beads were washed, and eluted proteins were detected by RT-qPCR analysis.

\section{RIP analysis}

RIP analysis was conducted in glioma cells using Magna RIP RNA-binding protein immunoprecipitation kit (Millipore, MA) according to manufacturer's instructions. Briefly, cells were collected after washing with cold PBS and RIP lysis buffer was added. The suspension was then centrifuged and $100 \mu \mathrm{L}$ from each cell lysate was transferred to the RIP immunoprecipitation buffer, which contained Ago2-conjugated magnetic beads and IgG as a negative control (Millipore, MA, USA). The magnetic beads were washed with RIP wash buffer and then incubated with proteinase $\mathrm{K}$ at $55^{\circ} \mathrm{C}$ for $30 \mathrm{~min}$. Subsequently, RNA was extracted for RT-qPCR analysis.

\section{In vivo xenograft experiments}

Male BALB/c nude mice ( 6 weeks old, $n=6$ ) were purchased from Beijing HFK Bioscience Co. Ltd. (Beijing, China) and were maintained under pathogen-free conditions. Animal experiments were approved by the Animal Care and Use Committee of The Second Affiliated Hospital of Zhejiang University School of Medicine and were performed in accordance with the relevant guidelines and regulations of the committee. For analysis of tumor propagation, $1 \times 10^{7} \mathrm{U} 251$ tumor cells, transfected with a short hairpin RNA (shRNA) targeting either LINC00174 (shLINC00174) or a negative control shRNA (shNC), were subcutaneously injected into BALB/c nude mice. Tumors were weighed 3 weeks after injection. Tumor volume was calculated at the indicated time points using the following formula: volume $=\pi \mathrm{ab}^{2} / 6$ (a, tumor length; b, tumor width). Ki67 levels were detected by immunohistochemical staining of tumors.

\section{Western blot analysis}

Total protein lysates were resolved by $10 \%$ SDS-PAGE and transferred to polyvinyl difluoride membranes (EMD Millipore, Billerica, MA, USA). Following blocking with 5\% nonfat dry milk in Tris-buffered saline containing $0.1 \%$ Tween-20 (TBS-T) for $30 \mathrm{~min}$ at $37^{\circ} \mathrm{C}$, membranes were washed four times in TBS-T and incubated with primary antibodies overnight at $4{ }^{\circ} \mathrm{C}$. The primary anti-bodies: antiSLC2A1 (Abcam, Cambridge, UK, ab190163, dilution: 1: 1000), E-cadherin (Abcam, ab15148, dilution: 1:1000), Ncadherin (Abcam, ab202030, dilution: 1:1000), Vimentin (Abcam, ab8978, dilution: 1:800), Cleaved caspase-3 (Abcam, ab2302, dilution: 1:800), Cleaved caspase-9 (Abcam, ab2324, dilution: 1:1000), Bcl-2 (Abcam, ab32124, dilution: 1:800), and Bax (Abcam, ab32503, dilution: 1:800) were used. Following extensive washing, membranes were incubated with a horseradish peroxidase-conjugated goat polyclonal anti-rabbit IgG secondary antibody (cat. no. 7074; Cell Signaling Technology, Danvers, MA, USA), at a dilution of 1:2000, for $1 \mathrm{~h}$ at room temperature. Immunoreactivity was detected by enhanced chemiluminescence (Pierce; Thermo Fisher Scientific, Inc., Waltham, MA, USA) and visualized using a ChemiDoc XRS imaging system and analysis software (Bio-Rad Laboratories, Inc., Hercules, CA, USA). $\beta$-actin (Abcam, ab179467, dilution: 1: $800)$ served as a loading control.

\section{Statistical analysis}

All data are presented as the mean \pm standard deviation (SD) from three independent experiments. The statistical analyses were performed by using SPSS 18.0 software (IBM, New York, USA). Differences between groups were analyzed by using Student's t-test (two groups) or one-way ANOVA (multiple groups). Overall survival (OS) was defined either as the time from surgery to death, or the time from surgery to the date of the last recorded follow-up visit. A Kaplan-Meier curve was plotted for survival analysis, and the difference between the two groups was compared using a log-rank test. Spearman's correlation analysis was used to determine the correlations between the levels of miR-152-3p and LINC00174/SLC2A1 in glioma tissues. $P<0.05$ was considered statistically significant.

\section{Results}

\section{LINC00174 was over-expressed in glioma tissues and cell} lines

We first identified the expression of LINC00174 in glioma tissues and normal tissues in 45 paired samples by RT-qPCR. The results showed that LINC00174 expression was increased significantly in glioma tissues compared with that in PTBE (Fig. 1a, $P<0.001$ ). The expression of LINC00174 in different stages of glioma samples was examined by RT-qPCR and ISH analysis. 
A

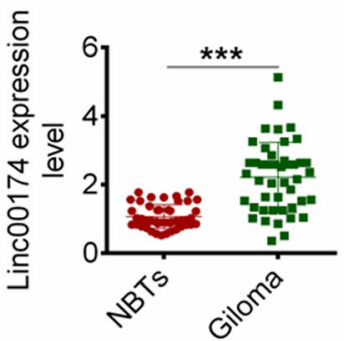

C

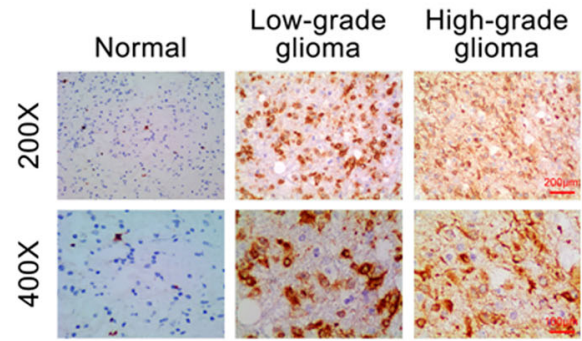

B
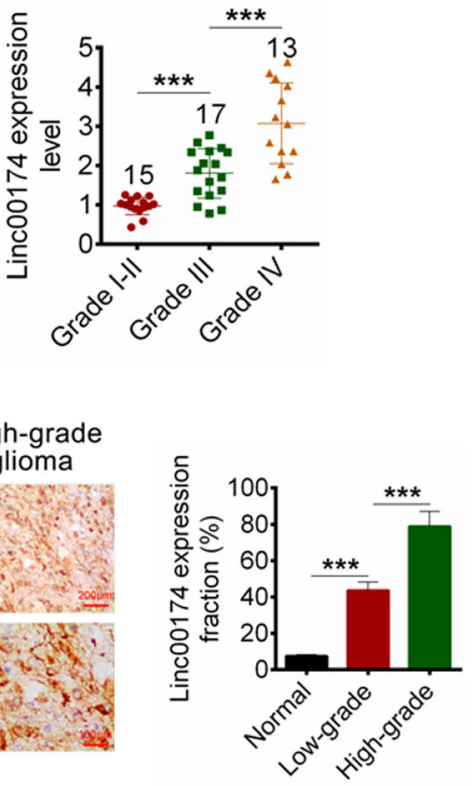

E

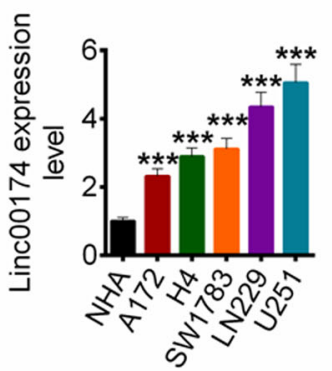

Fig. 1 The expression of LINC00174 in glioma tissues and cell lines. a The expression of LINC00174 in PTBE and glioma tissues was identified by RT-qPCR. b LINC00174 expression in different grades of glioma patients was examined by RT-qPCR. c ISH was used for the LINC00174 expression detection in normal tissue, low-grade and high-grade of glioma tissues. $\mathbf{d}$ Sunvival rates of patients with glioma with high and low LINC00174 by Kaplan-Meier survival analysis. e The expression of LINC00174 in glioma cell lines and NHA cells was examined by RT-qPCR. Data are presented as the mean \pm SD. ***P<0.001 vs. NHA cells

As shown in Fig. 1b-c, the LINC00174 expression was higher in high-grade than that in low-grade. Furthermore, the high expression of LINC00174 predicted an unfavourable prognosis (Fig. 1d). The expression of LINC00174 in human astrocytes (NHA) and five glioma cell lines including U251, LN229, H4, SW1783, and A172 was also examined. The results showed that LINC00174 was overexpressed in glioma cell lines (Fig. 1e, $P<0.001$ ).

\section{LINC00174 promoted cell proliferation of glioma cells in vivo and in vitro}

To identify the effect of LINC00174 on cell proliferation of glioma cells, U251 and LN229 cells were transfected with pcDNA3.1-LINC00174 or pLKO.1-LINC00174, or their relative controls. The transfection efficiency was then identified by RT-qPCR, and pLKO.1-LINC00174\#1 with better efficiency for LINC00174 knockdown was used for the further studies (Fig. 2a-b, $P<0.001$ ). Cell proliferation and apoptosis were then identified by CCK 8 and Tunel, respectively. As shown in Fig. 2c-d, cell proliferation of U251 and LN229 cells with pcDNA3.1-LINC00174 transfection was promoted compared with that of pcDNA3.1 transfected cells $(P<0.001)$, and pcDNA3.1-LINC00174 also decreased cell apoptosis of glioma cells $(P<0.001)$. On the contrary, pLKO.1-LINC00174 transefection significantly inhibited cell proliferation and facilitated cell apoptosis of U251 and LN229 cells compared with that of pLKO.1 transfected cells (Fig. 2c-d, $P<0.001$ ). Moreover, the effect of LINC00174 knockdown on tumor growth was also examined by a 


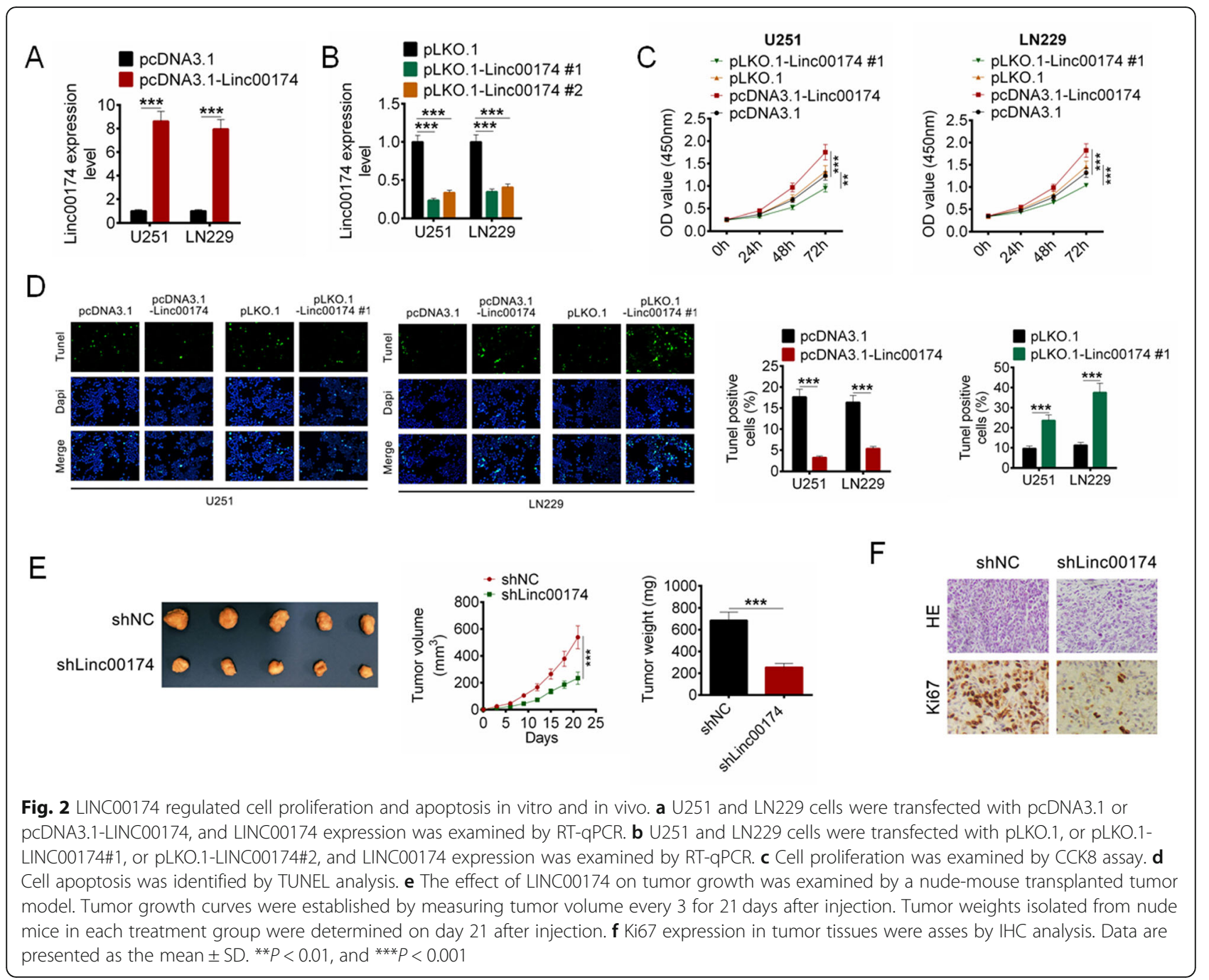

nude-mouse transplanted tumor model. The results exhibited that shLINC00174 obviously delayed tumor growth, decreased tumor volume, and reduced tumor weight compared with the shNC group (Fig. 2e, $P<0.001$ ). The LINC0074 knockdown also effectively inhibited the expression of Ki67 in tumor tissues in comparison with that in tumor tissues of shNC group (Fig. 2f, $P<0.001$ ).

\section{LINC00174 accelerated cell migration, invasion and glycolysis of glioma cells}

To explore the effect of LINC00174 on malignant tumor cell phenotype of glioma, cell migration, invasion and glycolysis of U251 and LN229 cells with LINC00174 overexpression or LINC00174 knockdown were evaluated. We found that LINC00174 overexpression promoted cell migration and invasion of U251 and LN229 cells, and LINC00174 knockdown inhibited cell migration and invasion of glioma cells (Fig. 3a-b, $P<0.001$ ). The effect of LINC00174 on glucose consumption and lactate production in U251 and LN229 cells was also identified. As shown in Fig. 3c, LINC00174 overexpression promoted the glucose consumption and lactate production $(P<0.001)$, while LINC00174 knockdown showed the opposite effect $(P<0.001)$.

\section{LINC00174 directly targeted miR-152-3p}

To further explore the underlying mechanism by which LINC00174 facilitated cell proliferation, migration, invasion and glycolysis of U251 and LN229 cells, the targeted miRNAs of LINC00174 were predicted. By FISH analysis in Fig. 4a, the expression of LINC00174 mainly located in the cytoplasm. From the analysis of Starbase (http://starbase.sysu.edu.cn), miR-152-3p was predicted to combine with LINC00174, which was verified by luciferase reporter assay (Fig. 4b-c). Furthermore, immunoprecipitation was carried out to verify the bioinformatical prediction. Also, RIP assay was performed to unveil whether LINC00174 interact miR-152-3p directly. As shown in Fig. 4d, the enrichment levels of LINC00174 and miR-152-3p in Ant-Ago2 group were higher than that in 
A
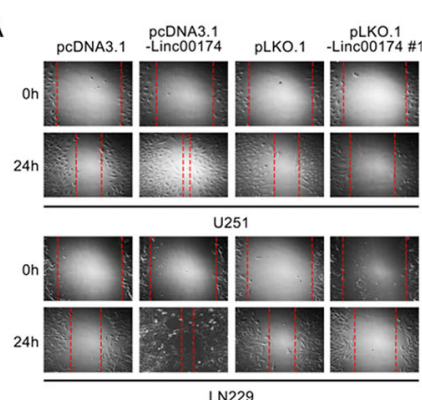

C

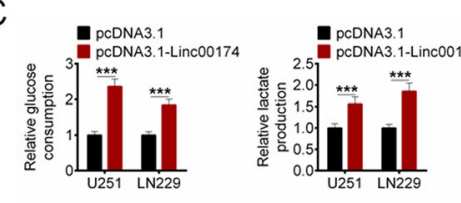

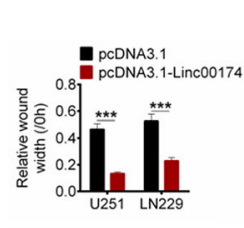
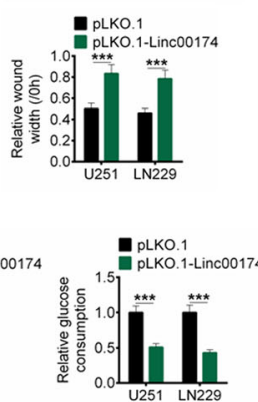

B
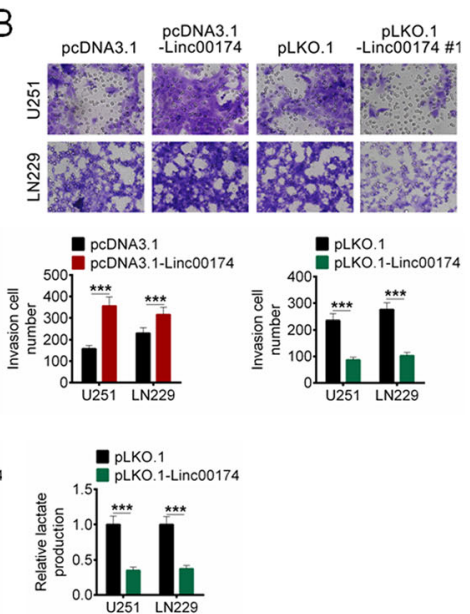

Fig. 3 LINC00174 regulated cell migration, invasion and glycolysis of glioma cells. a The effect of LINC00174 on cell migration of glioma cells was evaluated by wound healing assay. $\mathbf{b}$ Cell invasion of glioma cells was identified by transwell assay. c Glucose consumption and lactate production in U251 and LN229 cells with LINC00714 overexpression or knockdown were detected by ELISA analysis. Data are presented as the mean \pm SD. ${ }^{* * *} P<0.001$

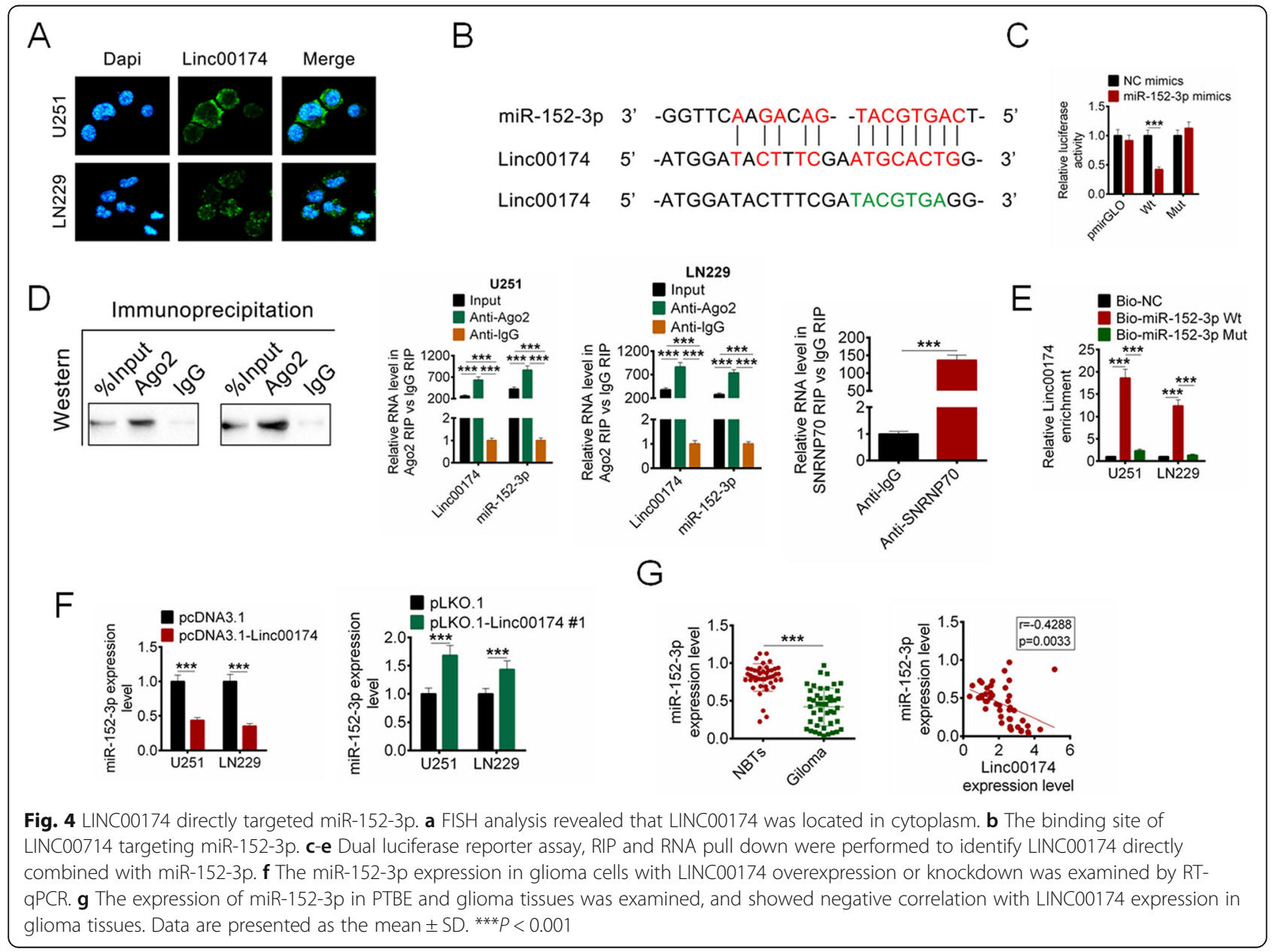


Ant-IgG group, the results suggested that miR-152-3p could directly target LINC00174 $(P<0.001$, Fig. $4 d)$. By RNA pull down analysis, the LINC00174 enrichment in Bio-miR-152-3p-MUT showed no significance compared with the Bio-NC, while the LINC00174 enrichment in Bio-miR-152-3p-WT was significantly increased compared with the the Bio-NC $(P<0.001$, Fig. $4 \mathrm{e})$. The expression of miR-152-3p in U251 and LN229 cells with pcDNA3.1-LINC00174, or pLKO.1-LINC00174 or the relative controls transfection was examined by RT-qPCR. The results showed that miR-152-3p expression was decreased in U251 and LN229 cells transfected with pcDNA3.1-LINC00174, while increased in U251 and LN229 cells transfected with pLKO.1-LINC00174 $(P<$ 0.001 , Fig. 4f). Moreover, the expression of miR-152-3p in glioma tissues and PTBE was identified. The expression of miR-152-3p in glioma tissues was decreased significantly compared with that in the PTBE, and the expression of which exerted a negative correlation with LINC00174 expression in glioma samples $(P<0.001$, Fig. $4 \mathrm{~g})$.

\section{miR-152-3p inhibited cell proliferation, migration and invasion through regulating SLC2A1 expression}

We then explored the target mRNA of miR-152-3p through bioinformatics analysis. From the Targetscan (http://www.targetscan.org/vert_71/) analysis, SLC2A1 was predicted as the target of miR-152-3p (Fig. 5a). The interaction between miR-152-3p and SLC2A1 was then examined by luciferase reporter assay (Fig. 5b). U251 and LN229 cells were then transfected with miR-152-3p mimics or miR-152-3p inhibitors, and the transfection efficiency was evaluated by RT-qPCR $(P<0.001$, Fig. $5 c)$. The mRNA expression and protein level of SLC2A1 in U251 and LN229 cells with miR-152-3p mimics or miR$152-3 p$ inhibitors transfection were identified by RTqPCR and western blot, and the results showed that SLC2A1 expression was decreased in glioma cells with miR-152-3p mimics transfection, and increased in glioma cells with miR-152-3p inhibiors transfection $(P<$ 0.001, Fig. 5d-e). Furthermore, SLC2A1 expression was increased in glioma tissues and negatively associated with miR-152-3p expression in glioma samples $(P<$ 0.001, Fig. 5f).

The rescued experiments were performed, U251 cells were transfected with NC mimics, or miR-152-3p mimics, or miR-152-3p mimics + pcDNA3.1, or miR152-3p mimics + pcDNA3.1-SLC2A1. SLC2A1 expression was increased in U251 cells with miR-152-3p mimics + pcDNA3.1-SLC2A1 transfection $(P<0.001$, Fig. 5g). Cell proliferation, apoptosis, migration, invasion and glycolysis were then identified. As shown in Fig. 5hl, cell proliferation, migration, invasion and glycolysis of U251 cells was significantly inhibited by miR-152-3p mimics compared with the NC mimics group $(P<$
0.001), cell apoptosis was increased in U251 cells transfected with miR-152-3p mimics $(P<0.001)$. While SLC2A1 overexpression rescued the anti-tumor effect of miR-152-3p on U251 cells $(P<0.001$, Fig. 5 h-l).

\section{LINC00174 exerted the oncogenesis role in glioma via targeting miR-152-3p}

To further identify the underlying mechanism by which LINC00174 regulated cell activities in glioma cells, rescue assays were performed to further confirm that LINC00174 contributed to glioma progression by regulating miR-1523p/SLC2A1 signal pathway. U251 cells were transfected with pLKO.1, or pLKO.1-LINC00174\#1, or pLKO.1LINC00174\#1 + miR-NC, or pLKO.1-LINC00174\#1 + miR-152-3p inhibitor. Cell proliferation, apoptosis, migration, invasion and glycolysis were then identified by CCK8, TUNEL, wound healing, transwell and ELISA, respectively. It was found that SLC2A1 expression was increased in U251 cells with pLKO.1-LINC00174\#1 + miR152-3p inhibitor transfection $(P<0.001$, Fig. 6a). as shown in Fig. 6b-f, LINC00174 knockdown effectively inhibited cell proliferation, migration, invasion and glycolysis, and increased cell apoptosis of U251 cells compared with the cells with pLKO.1 tranfection, and miR-152-3p inhibitor transfection significantly reversed the inhibiting effect of LINC00174 knockdown on U251 cells $(P<0.001$, Fig. 6bf). Besides, the expressions of SLC2A1, E-cadherin, N-cadherin, Vimentin, Cleaved caspase-3, Cleaved caspase-9, Bcl-2, and Bax were identified by western blot analysis. LINC00174 knockdown evidently regulated the protein expression, while miR-152-3p inhibitor effectively abolished the effect of LINC00174 knockdown on protein expression (Fig. 6g).

\section{Discussion}

Gliomas are the most common malignant tumors in the central nervous system, and the exact pathogenesis of which is still unclear. LncRNA is a kind of non-proteincoding RNA, which plays an indispensable role in the occurrence and development of various tumors. Shen et al. reported that IncRNA LINC00174 was overexpressed in colorectal cancer samples and cells, and abnormal expression of LINC00174 indicated a poor prognosis of colorectal cancer patients. In the present study, we first identified the expression of LINC00174 in the glioma tissues and cells. We found that LINC00174 was overexpressed in the glioma tissues and cells, and high expression of LINC00174 showed a unfavourable prognosis in glioma patients. The effect of LINC00174 on cell proliferation, apoptosis, migration and invasion was also examined, and the results exhibited that LINC00174 knockdown effectively inhibited cell proliferation, migration and invasion, and promoted cell apoptosis of U251 and LN229 cells. Furthermore, LINC00174 


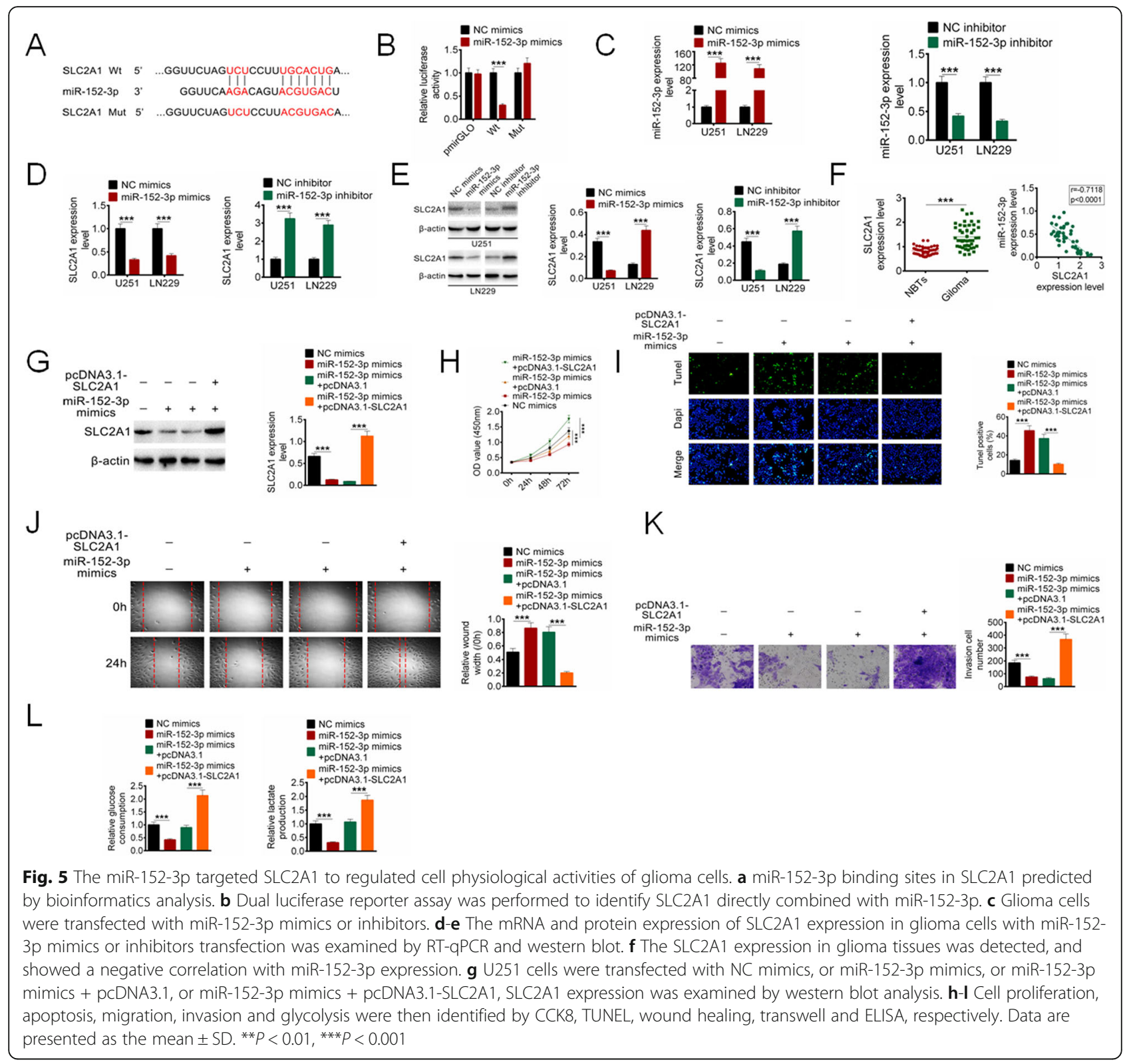

overexpression accelerated cell proliferation, migration and invasion, and decreased cell apoptosis of U251 and LN229 cells. In addition, the silencing LINC00174 could delay tumor growth in vivo. These data reveals that LINC00174 acts as an oncogene in glioma and facilitates the progression of glioma.

We subsequently explored the targeted miRNA of LINC00174 by biological analysis, and miR-152-3p was predicted to combine with LINC00174, which was also verified by RIP, RNA pull down and dual luciferase reporter analysis. A number of studies reported that miR152-3p expression was decreased in glioma [22], breast cancer [23], hepatic carcinoma [24], malignant melanoma [25], and prostate cancer [26]. Overexpression of miR152-3p showed anti-tumor effect on cancer cells [26]. Sun et al. reported that miR-152-3p was down-regulated in glioma samples and inhibited cell proliferation and invasion by suppressing the expression of DNMT1 [22]. In the present study, we found that miR-152-3p could interact with LINC00174, and miR-152-3p expression was negatively correlated with LINC00174 expression in glioma clinical samples. Moreover, siLINC00174 attenuated cellular activities of glioma cells, while miR-152-3p inhibitors evidently reversed the anti-tumor effect of siLINC00174 on glioma cells. The above results investigate that LINC00174 regulates cell phenotype of glioma cells via targeting miR-152-3p.

The target mRNA of miR-152-3p was afterwards examined. SLC2A1 was the downstream target of miR152-3p. SLC2A1 is a ratelimiting transporter for glucose 


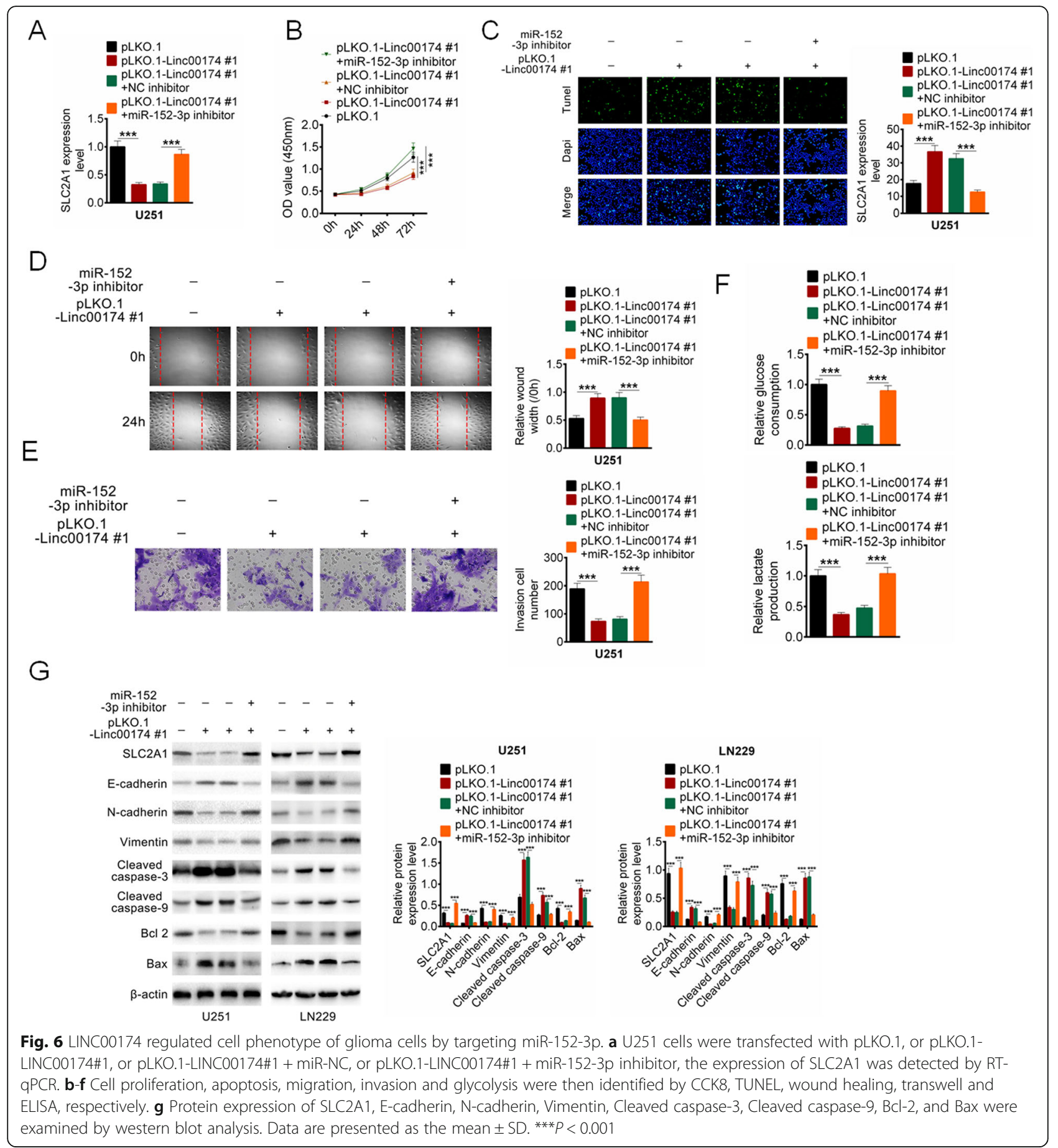

uptake, and plays a crucial role in glycolysis. Cancer cells characterized by rapid proliferation require more energy produced by glycolysis than normal cells. Previous studies reported that SLC2A1 expression was up-regulated in non-small cell lung cancer [27], colon cancer [28], and gastric cancer [20], and mediated the glucose transport in cancer cells. Chen et al. reported that cAMP responsive element binding protein 1 affected glucose transport in glioma cells by regulating the expression of
GLUT1 (SLC2A1), and mediated the metabolism and progression of glioma [29]. In this work, it was found that SLC2A1 was overexpressed in glioma samples, and SLC2A1 expression was negatively associated with miR152-3p expression in glioma patients. By a series of cellular functional experiments, we demonstrated that LINC00174 could promote the glycolysis in glioma cells. To further identify whether LINC00174 facilitates the glycolysis by regulating miR-152-3p/SLC2A1 pathway, 
the rescue experiments were performed. The results displayed that miR-152-3p mimic inhibited cell proliferation, migration, invasion and glycolysis in glioma cells, while SLC2A1 knockdown abolished the effect of miR152-3p mimic on glioma cells. The results reveal that LINC00174 promotes glioma cell proliferation, migration, invasion and glycolysis through regulating miR152-3p/SLC2A1 axes. Furthermore, the expressions of SLC2A1, E-cadherin, N-cadherin, Vimentin, Cleaved caspase-3, Cleaved caspase-9, Bcl-2, and Bax were identified by western blot analysis. E-cadherin, N-cadherin, and Vimentin are important factors participating in the epithelial-mesenchymal transition (EMT), which promotes the migration and invasion of cells [30]. As well known, Cleaved caspase-3, Cleaved caspase-9, Bcl-2, and Bax play crucial role in cell apoptosis [31]. LINC00174 knockdown evidently regulated the protein expression, while miR-152-3p inhibitor effectively abolished the effect of LINC00174 knockdown on protein expression. The results indicate that LINC00174 adjust cellular activities by regulating these proteins.

The highlights of our study are: (1) LINC00174 was overexpressed in glioma. (2) LINC00174 predicted an unfavorable prognosis in glioma patients. (3) LINC00174 promoted glycolysis and tumor progression by targeting miR-152-3p/SLC2A1 axes. Although a lot of studies demonstrates that SLC2A1 mediated the glucose transport in cancer cells, few studies focus on the detail function and the mechanism of SLC2A1 in glioma, and the pathways related with SLC2A1 in cancer progression are also rarely studied. Systematic study concerning the biological function and mechanism of SLC2A1 in glioma will be an important part of our future studies.

\section{Conclusions}

In general, our study investigated that LINC00174 expression was increased in glioma tissues and cell lines. High expression of LINC00174 predicted an unfavorable prognosis in glioma patients. LINC00174 promoted cell proliferation, migration, invasion and glycolysis via sponging miR-152-3p and up-regulating SLC2A1 expression. These results and findings provide a novel insight for glioma diagnosis and treatment.

\section{Abbreviations \\ CCK-8: Cell Counting Kit-8; CRC: Colorectal cancer; EMT: Epithelial- mesenchymal transition; FBS: Fetal bovine serum; GLUT1: Glucose transporter 1; IncRNA: Long-chain non-coding RNA; NHAs: Normal human astrocytes; PTBE: Peritumoral brain edema; RT-qPCR: Reverse transcription-quantitative polymerase chain reaction; shRNA: Short hairpin RNA; siRNA: Small interfering RNAs; SLC2A1: Solution vector family 2 promotes glucose transporter 1; TCGA: The Cancer Genome Atlas}

\section{Acknowledgements}

We thank the Department of Pathology of The Second Affiliated Hospital of Zhejiang University School of Medicine.

\section{Authors' contributions}

JS and XZ designed the study. JS and YZ collected the clinical data and performed the experiments. $B Q$ and $Y W$ performed the animal study and data analysis. JS and XZ wrote the manuscript. All authors read and approved the final manuscript.

\section{Funding}

The study was supported by Natural Science Foundation of Zhejiang Province (LY16H090005), China.

\section{Availability of data and materials \\ The data used in this study is available on a reasonable request from the corresponding author.}

\section{Ethics approval and consent to participate}

The present study was approved by the Institutional Ethical Review Committee of The Second Affiliated Hospital of Zhejiang University School of Medicine.

\section{Consent for publication}

Not applicable.

\section{Competing interests}

The authors declare that they have no competing interests.

Received: 2 July 2019 Accepted: 26 August 2019

Published online: 06 September 2019

\section{References}

1. Miyauchi JT, Tsirka SE. Advances in immunotherapeutic research for glioma therapy. J Neurol. 2018;265(4):741-56.

2. Wang $X$, Qiu Y, Yu Q, et al. Enhanced glioma therapy by synergistic inhibition of autophagy and tyrosine kinase activity. Int J Pharm. 2018; 536(1):1-10.

3. Ostrom QT, Cote DJ, Ascha M, Kruchko C, Barnholtz-Sloan JS. Adult glioma incidence and survival by race or ethnicity in the United States from 2000 to 2014. JAMA Oncol. 2018;4(9):1254-62.

4. Liang M, Gao C, Wang Y, et al. Enhanced blood-brain barrier penetration and glioma therapy mediated by T7 peptide-modified low-density lipoprotein particles. Drug Deliv. 2018;25(1):1652-63.

5. Wang F, Zheng Z, Guan J, et al. Identification of a panel of genes as a prognostic biomarker for glioblastoma. EBioMedicine. 2018;37:68-77.

6. Guo Q, Guan GF, Cheng W, et al. Integrated profiling identifies caveolaeassociated protein 1 as a prognostic biomarker of malignancy in glioblastoma patients. CNS Neurosci Ther. 2019;25(3):343-54.

7. Zhang Z, Liu Q, Wang P, et al. Development and internal validation of a nine-IncRNA prognostic signature for prediction of overall survival in colorectal cancer patients. PeerJ. 2018;6:e6061.

8. Zhao $\mathrm{K}$, Jin $\mathrm{S}$, Wei B, Cao S, Xiong Z. Association study of genetic variation of IncRNA MALAT1 with carcinogenesis of colorectal cancer. Cancer Manag Res. 2018;10:6257-61.

9. Huang $P$, Li F, Li L, et al. IncRNA profile study reveals the mRNAs and IncRNAs associated with docetaxel resistance in breast cancer cells. Sci Rep. 2018;8(1):17970.

10. Deng SJ, Chen HY, Zeng Z, et al. Nutrient stress-dysregulated antisense IncRNA GLS-AS impairs GLS-mediated metabolism and represses pancreatic cancer progression. Cancer Res. 2019;79(7):1398-412.

11. Xiong Z, Wang L, Wang Q, Yuan Y. LncRNA MALAT1/miR-129 axis promotes glioma tumorigenesis by targeting SOX2. J Cell Mol Med. 2018;22:3929.

12. Lu YF, Cai XL, Li ZZ, et al. LncRNA SNHG16 functions as an oncogene by sponging MiR-4518 and up-regulating PRMT5 expression in glioma. Cell Physiol Biochem. 2018;45(5):1975-85.

13. Liang C, Yang Y, Guan J, et al. LncRNA UCA1 sponges miR-204-5p to promote migration, invasion and epithelial-mesenchymal transition of glioma cells via upregulation of ZEB1. Pathol Res Pract. 2018;214(9):1474-81.

14. Shen Y, Gao X, Tan W, XU T. STAT1-mediated upregulation of IncRNA LINC00174 functions a ceRNA for miR-1910-3p to facilitate colorectal carcinoma progression through regulation of TAZ. Gene. 2018;666:64-71.

15. Sun J, Tian X, Zhang J, et al. Regulation of human glioma cell apoptosis and invasion by miR-152-3p through targeting DNMT1 and regulating NF2: 
MiR-152-3p regulate glioma cell apoptosis and invasion. J Exp Clin Cancer Res. 2017;36(1):100.

16. Zhang $P$, Sun $H$, Yang $B$, et al. miR-152 regulated glioma cell proliferation and apoptosis via Runx2 mediated by DNMT1. Biomed Pharmacother. 2017;92:690-5.

17. Nazim B, Pascal B, Henri S. GLUT1: A novel tool reflecting proliferative activity of lung neuroendocrine tumors? Pathol Int. 2017;67:32-6.

18. Zibo Z, Lu W, Elizabeth B, et al. $\beta$-Catenin/Tcf7/2-dependent transcriptional regulation of GLUT1 gene expression by Zic family proteins in colon cancer. Sci Adv. 2019;5:eaax0698.

19. Ooi AT, Gomperts BN. Molecular pathways: targeting cellular energy metabolism in cancer via inhibition of SLC2A1 and LDHA. Clin Cancer Res. 2015;21(11):2440-4.

20. Ding X, Liu J, Liu T, Ma Z, Wen D, Zhu J. miR-148b inhibits glycolysis in gastric cancer through targeting SLC2A1. Cancer Med. 2017;6(6):1301-10.

21. Santasusagna S, Moreno I, Navarro A, et al. miR-328 mediates a metabolic shift in colon cancer cells by targeting SLC2A1/GLUT1. Clin Transl Oncol. 2018;20(9):1161-7.

22. Zhang $P$, Sun $H$, Yang B, et al. miR-152 regulated glioma cell proliferation and apoptosis via Runx2 mediated by DNMT1.Biomed. Pharmacother. 2017; 92:690-5.

23. Marques J, Mota AL, Oliveira JG, et al. Melatonin restrains angiogenic factors in triple-negative breast cancer by targeting miR-152-3p: in vivo and in vitro studies. Life Sci. 2018;208:131-8.

24. Yin T, Liu MM, Jin RT, Kong J, Wang SH, Sun WB. miR-152-3p modulates hepatic carcinogenesis by targeting cyclin-dependent kinase 8. Pathol Res Pract. 2019:215:152406

25. Luan W, Li R, Liu L, et al. Long non-coding RNA HOTAIR acts as a competing endogenous RNA to promote malignant melanoma progression by sponging miR-152-3p. Oncotarget. 2017:8(49):85401-14.

26. Moya L, Meijer J, Schubert S, Matin F, Batra J. Assessment of miR-98-5p, miR152-3p, miR-326 and miR-4289 expression as biomarker for prostate cancer diagnosis. Int J Mol Sci. 2019;20(5):1154.

27. Kim SJ, Hwang SH, Kim IJ, et al. The association of 18F-deoxyglucose (FDG) uptake of PET with polymorphisms in the glucose transporter gene (SLC2A1) and hypoxia-related genes (HIF1A, VEGFA, APEX1) in non-small cell lung cancer. SLC2A1 polymorphisms and FDG-PET in NSCLC patients. J Exp Clin Cancer Res. 2010;29:69.

28. Goos JA, de Cuba EM, Coupe VM, et al. Glucose transporter 1 (SLC2A1) and vascular endothelial growth factor a (VEGFA) predict survival after resection of colorectal cancer liver metastasis. Ann Surg. 2016;263(1):138-45.

29. Chen J, Zhang C, Mi Y, Chen F, Du D. CREB1 regulates glucose transport of glioma cell line U87 by targeting GLUT1. Mol Cell Biochem. 2017;436(1-2):79-86.

30. Wang J, Zhou F, Li Y, et al. Cdc20 overexpression is involved in temozolomide-resistant glioma cells with epithelial-mesenchymal transition. Cell Cycle. 2017;16(24):2355-65.

31. Zhang Y, Sui R, Chen Y, Liang H, Shi J, Piao H. Long noncoding RNA MT1JP inhibits proliferation, invasion, and migration while promoting apoptosis of glioma cells through the activation of PTEN/Akt signaling pathway. J Cell Physiol. 2019;234(11):19553-64.

\section{Publisher's Note}

Springer Nature remains neutral with regard to jurisdictional claims in published maps and institutional affiliations.

Ready to submit your research? Choose BMC and benefit from:

- fast, convenient online submission

- thorough peer review by experienced researchers in your field

- rapid publication on acceptance

- support for research data, including large and complex data types

- gold Open Access which fosters wider collaboration and increased citations

- maximum visibility for your research: over $100 \mathrm{M}$ website views per year

At $\mathrm{BMC}$, research is always in progress.

Learn more biomedcentral.com/submissions 